\title{
Performance and Accuracy Investigations of Two Doppler Global Velocimetry Systems Applied in Parallel
}

\author{
Christian Willert ${ }^{1}$, Guido Stockhausen ${ }^{1}$, Joachim Klinner ${ }^{1}$, \\ Christine Lempereur ${ }^{2}$, Philippe Barricau², \\ Philippe Loiret $^{3}$, Jean Claude Raynal ${ }^{3}$
}

1: Institute of Propulsion Technology, German Aerospace Center (DLR), 51170 Koeln, Germany; Chris.Willert@dlr.de 2: Aerodynamics and Energetics Modeling Department, ONERA, 2 Avenue Edouard Belin, 31055 Toulouse Cedex, France; Christine.Lempereur@onecert.fr

3: DSFM/GFR, ONERA, Centre du Fauga Mauzac, 31410 Mauzac, France; Philippe.Loiret@onera.fr

\begin{abstract}
Two Doppler global velocimetry systems were applied in parallel to assess their performance in wind tunnel environments. Both DGV systems were mounted on a common traverse surrounding the glasswalled $1.4 \times 1.8 \mathrm{~m}^{2}$ test section of the wind tunnel. The traverse normally supports a three-component forward-scatter laser Doppler velocimetry system. The reproducible vortical flow field generated by the blunt tip of an airfoil was chosen for this investigation and was precisely surveyed by LDA just prior to the DGV measurements.

Both DGV systems shared the same continuous wave laser light source, laser frequency stabilization and fiber optic light sheet delivery system. The principle differences between the DGV implementations are with regard to the imaging configuration. One configuration relied on a single camera that observed three successively operated light sheets. In the second configuration, three camera views simultaneously observed a single light sheet using a four-branch fiber imaging bundle.

The imaging bundle system had all three view points in a forward scattering arrangement which increased the scattering efficiency but reduced the frequency shift sensitivity. Since all three light sheet observation components were acquired onto the same image frame, acquisition times could be reduced to a minimum. On the other hand, the triple light sheet - single camera system observed two light sheets in forward scatter and one light sheet in backscatter. Although three separate images had to be recorded in succession, the image quality, spatial resolution and signal-to-noise ratio was superior to the imaging bundle system.

Comparison of the DGV data with LDV measurements shows very good agreement to within 1-2 m/s. The remaining discrepancy has a variety of causes, some are related to the reduced resolving power of the fiber imaging bundle system (graininess, smoothing), exact localization of the receiver head with respect to the scene, laser frequency drift or background influences. The extensive data base available allows a more detailed study of the influences of each of these factors.
\end{abstract}

\section{Introduction}

Over the past decade planar flow field measurements in wind tunnels have gained increased importance in CFD-driven validation experiments as they are capable of characterizing the flow much more efficiently than scanning point-wise techniques such as five-hole probes or laserDoppler anemometry (LDA). In particular particle image velocimetry (PIV) has matured to become a well accepted flow diagnostic tool and already has found application in a large variety of facilities. Doppler global velocimetry (DGV), also known as planar Doppler velocimetry (PDV), provides the same planar velocity data as PIV but relies on completely different measurement principles (Elliot \& Beutner 1999, Saminy \& Wernet 2000, Willert 2005). In part due to the success of PIV, but also due to the increased system complexity, the DGV technique has not yet reached the level of maturity as PIV. At this point few research groups actively pursue the development of the technique.

Nonetheless DGV has a number of characteristics which allow flow field measurements where the PIV technique is hampered. While PIV relies on resolving discrete particles, DGV as an intensity-based technique will work in conditions with poor optical resolving power. This allows, 
for instance, the use of endoscopes to image internal duct flows or even in-cylinder piston engine flows. Optical blurring due to strong refractive index gradients, as found in pressurized combustion has little influence on the quality of the DGV measurements.

In the course of DGV development a variety of error sources have been identified. In effect DGV is an analog technique (intensity measurement) and therefore is very susceptible to stray light. Considerable effort and experience is required to minimize these contaminating effects. To further quantify the limitations of a given DGV system implementation requires experiments with clearly defined boundary conditions, preferably with reference measurements obtained by an alternative technique. The joint ONERA-DLR research project to be described in the following provided the unique opportunity to perform side-by-side measurements simultaneously on a reproducible wind tunnel flow using two different DGV system implementations. Accompanying LDA data served as a benchmark for comparison.
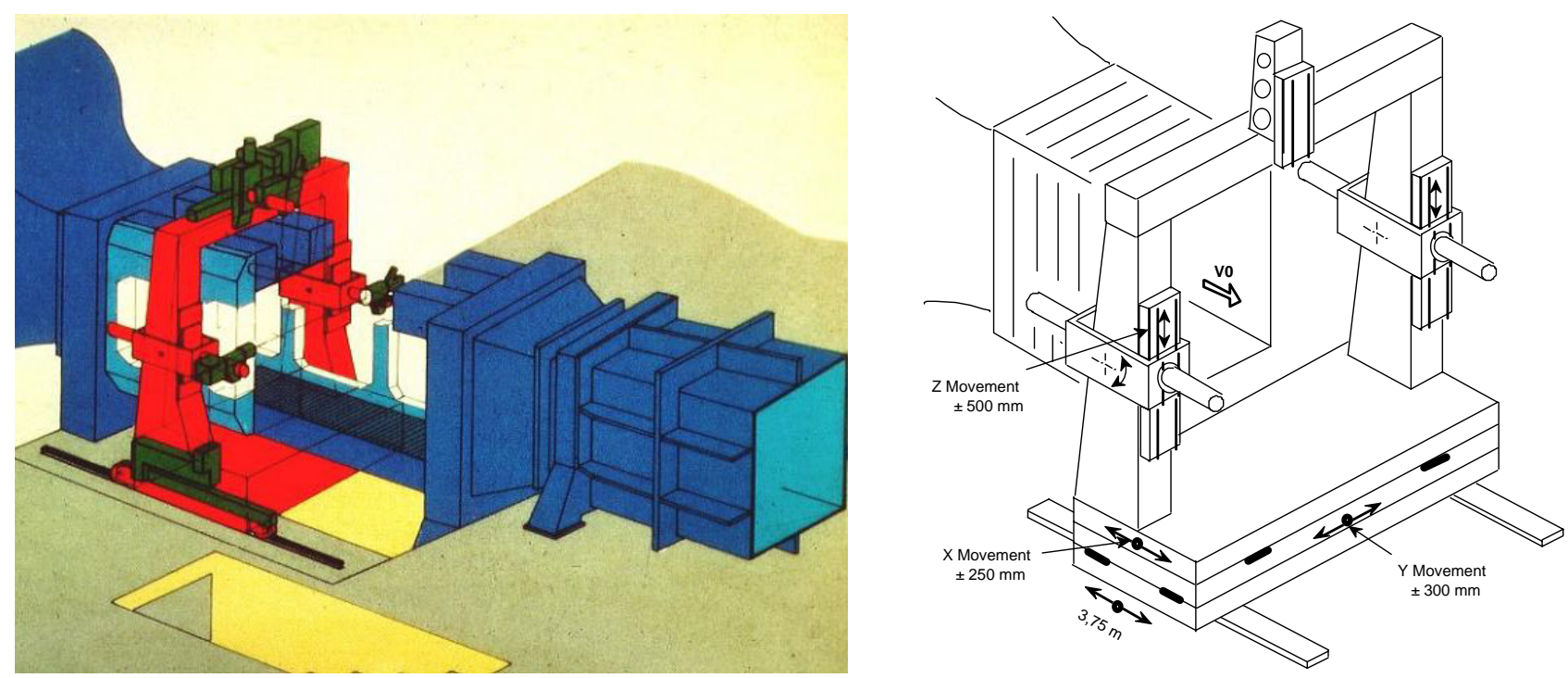

Fig. 1 Test section of ONERA-F2 wind tunnel (left) and LDA traverse system (right)

\section{Wind tunnel facility, model and seeding}

The ONERA F2 subsonic research wind tunnel was chosen mainly because of its large optical access. The side walls of the $5 \mathrm{~m}$ long test section $\left(1.4 \times 1.8 \mathrm{~m}^{2}, \mathrm{~W} \times \mathrm{H}\right)$ consist of large glass panels such that few restrictions are imposed regarding the positioning of optical instrumentation. The test section is surrounded by a traverse system ( $40 \mu \mathrm{m}$ accuracy) that normally supports the LDA optics that also could be used as a common support of the DGV emitting and receiving optics. A traversal along the stream-wise direction would therefore allow an investigation of the wake flow development without a need for recalibrating the DGV imaging configuration.

The tip vortex developing in the wake of a blunt-edged, symmetrical wing model (300 mm chord, $31.5 \mathrm{~mm}$ thickness) was chosen for this investigation. The airfoil was mounted on the center of the bottom floor and could be rotated to different angles of attack. The free-stream conditions were fixed at $68 \mathrm{~m} / \mathrm{s}$.

For the DGV measurements the flow was seeded with small oil droplets of $0.3-0.5 \mu \mathrm{m}$ diameter (according to manufacturer specifications). These droplets were generated with a smoke generator by means of evaporation and re-condensation of refined oil $\left(220^{\circ} \mathrm{C}\right.$ boiling point). A fan transported the seeding through a pipe into the wind tunnel facility upstream of the settling chamber. The pipe's exit was positioned to seed mainly the area of interest although long-lasting global seeding could be achieved after several minutes of continuous seeding injection due to the accumulation of seeding in the closed-circuit facility. 

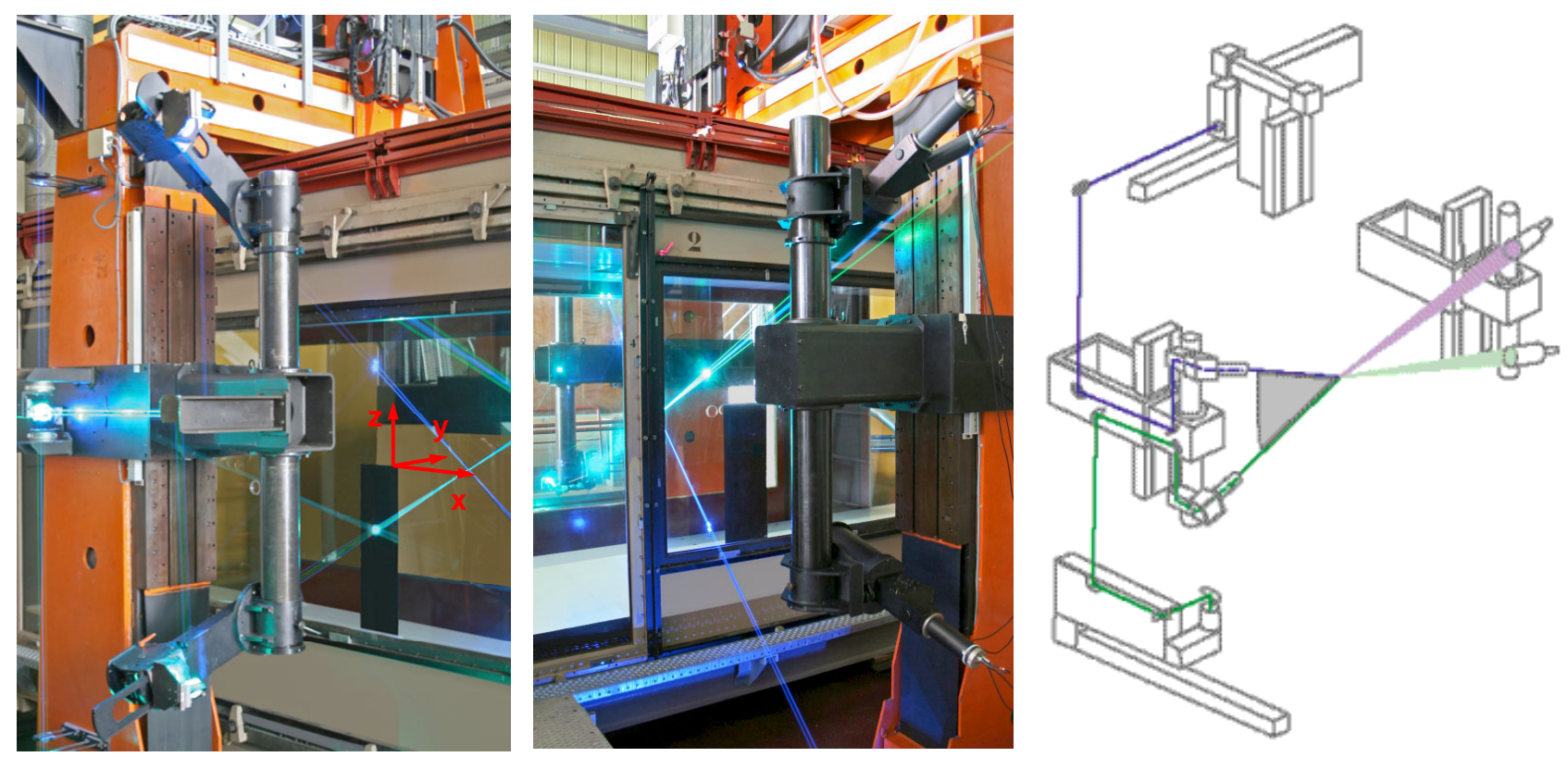

Fig. 2 LDA system mounted on traverse system: transmitting optics (left), receiving optics (middle). The airfoil model used for this investigation is visible in the middle of the test section.

\section{Instrumentation}

\subsection{LDA system}

As mentioned before, the test section is surrounded by a traversable gantry which supports a three-component LDA system (Fig. 2). The classical fringe mode LDA system is operated in forward scatter arrangement with receivers placed on the opposite side of the test section (Fig. 2, middle). The transmitting optics, supplied with light by a pair of argon-ion lasers, consist of a $f l=1500 \mathrm{~mm}$ head for the green $(514 \mathrm{~nm})$ and blue $(488 \mathrm{~nm})$ channels and a $f l=1750 \mathrm{~mm}$ head for the purple $(476.5 \mathrm{~nm})$ channel. The enclosed angle between the heads is $56^{\circ}$. The probe volume has a size of $300 \mu \mathrm{m}$ and is imaged by a pair of Cassegrain telescopes fitted with either one (purple) or two (green, blue) photomultipliers. The acquired Doppler signals are processed by a frequency domain burst detector system (TSI FSA4000) operated in coincidence mode with the acceptance window set at $5 \mu \mathrm{s}$.

The described LDA system was used to acquire the flow field in a plane $450 \mathrm{~mm}$ downstream of the airfoil (Fig. 3). The measurement grid has a resolution of $5 \times 5 \mathrm{~mm}^{2}$ with integration times on the order of 1-2 seconds per point during which 2000 samples were acquired. The entire flow map was acquired in about 2 hours. Flow seeding was based on incense smoke (size $<0.5 \mu \mathrm{m}$ ) that was introduced upstream of the settling chamber.

\subsection{DGV system installation}

The traversable gantry supporting the LDA system was also used to support the light delivery and imaging components of the DGV system installation (Fig. 4). Two DGV receiver camera systems were installed in parallel and shared a common field of view and a triple light sheet arrangement which allowed truly simultaneous, time-averaged DGV measurements. Singlefrequency laser light was provided by an argon-ion laser with 1-2W power at $514 \mathrm{~nm}$ (green light). Since the laser frequency was slowly drifting and not actively stabilized, a fiber-optic frequency monitoring system was used to provide a set of reference signals of known relative frequency separation to the cameras (Barricau et al. 2002, Lempereur et al. 2006). An opto-mechanical switching device allowed selective light delivery via fiber-optic lines (10 $\mu \mathrm{m}$ core, $10-15 \mathrm{~m}$ lengths) to one of the three light sheet generators operating on a scanning principle (Willert et al. 2005). 
The two DGV camera systems have a similar optical layout using a non-polarizing beam splitter to separate the optical paths onto two CCD cameras (1376 x 1040 pixels) with an iodine cell placed in the path of one imaging leg. Residual polarization sensitivity of the beam splitter is reduced by placing either a wedge-depolarizer or a continuously rotating half-wave plate in front of the beam splitters. Readers are referred to Lempereur et al. (2006) and Willert et al. (2005) for further details on the camera systems. In the current application one camera system images the field of view from a single position, while the other system relies on a fiber imaging bundle system to collect the signal from three different viewpoints simultaneously (Nobes et al. 2004, Willert et al. 2005). The fourth imaging bundle of the quadruple branch system is used to collect the laser reference signal. Thus the principle difference between the two DGV implementations lies in the method by which the Doppler shifted signal is collected using a combination of different light sheets and camera (viewing) positions. A performance analysis on these systems is described in the following.

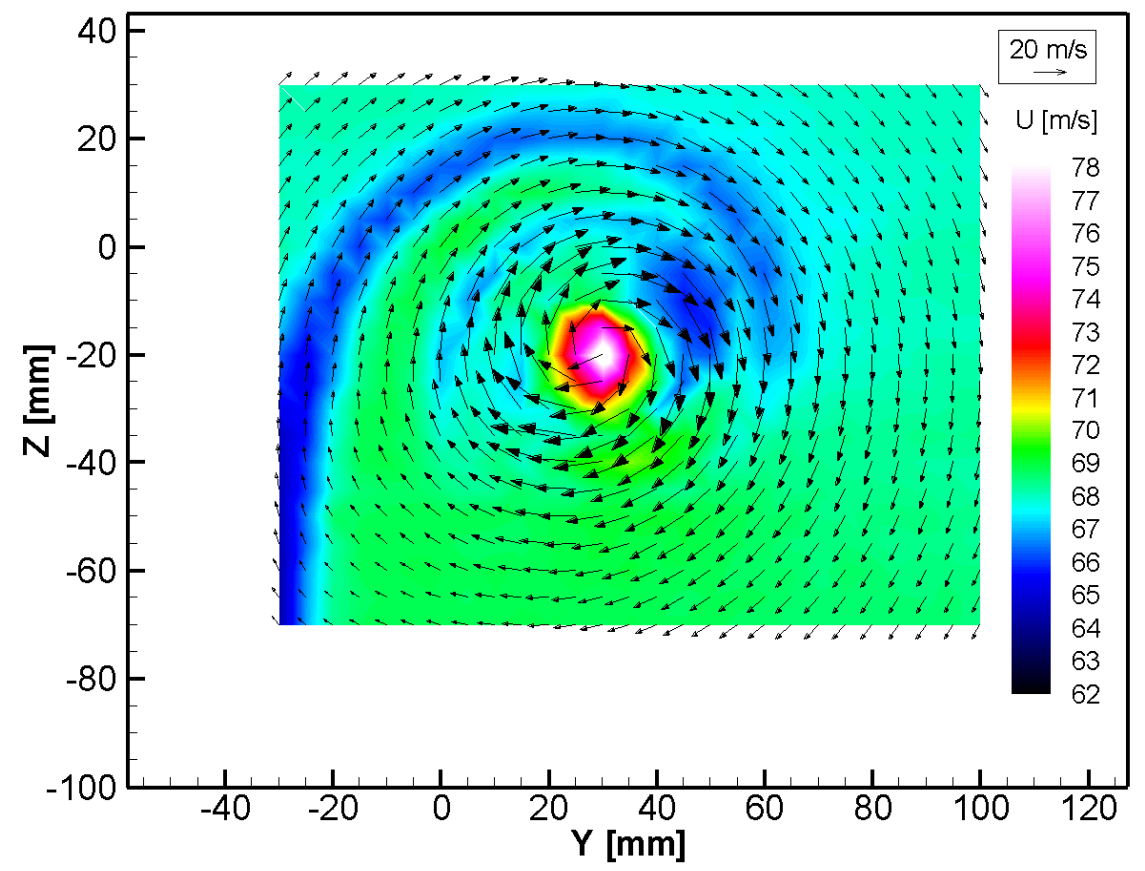

Fig. 3 Reference LDA measurements obtained downstream of an airfoil at $U_{\infty}=67 \mathrm{~m} / \mathrm{s}$ and angle of attack $\alpha=8.5^{\circ}$ using LDA system shown in Fig. 2.
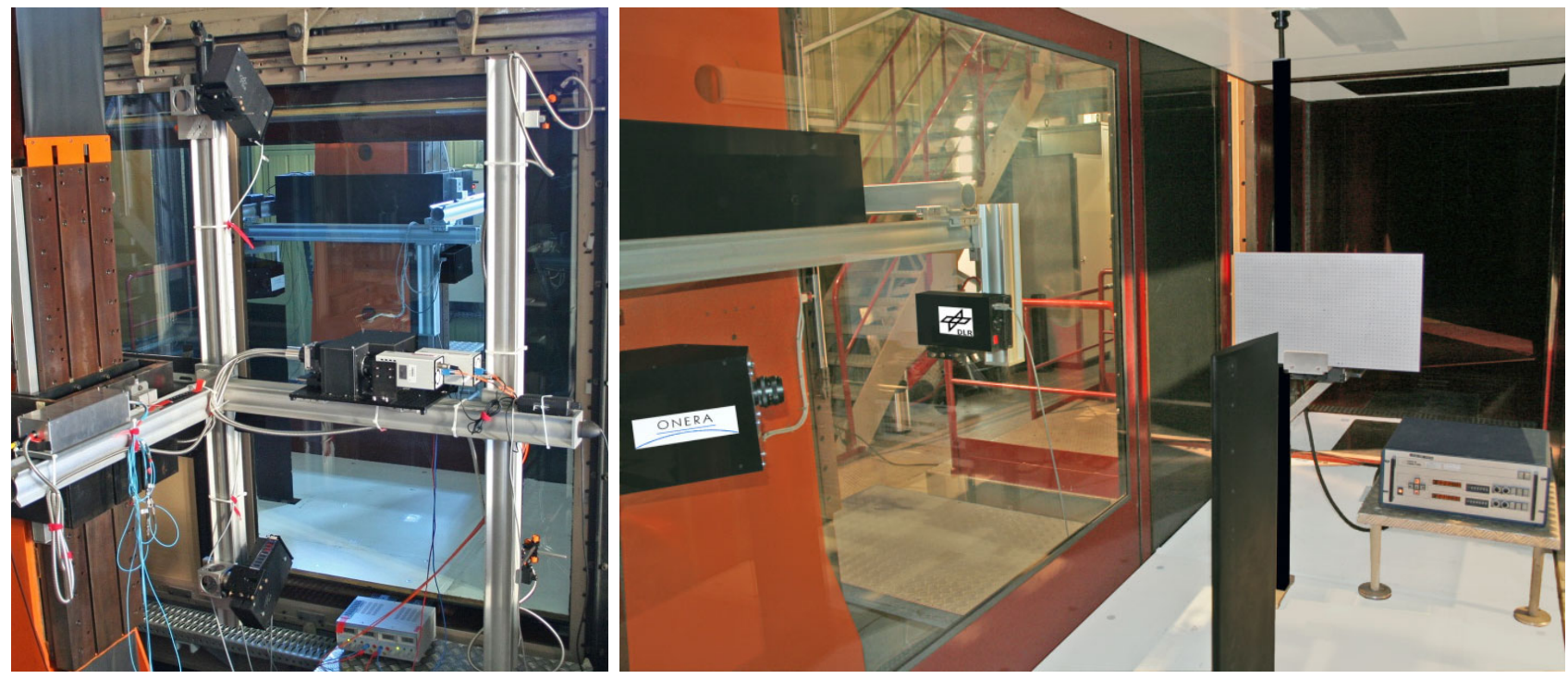

Fig. 4 DGV hardware installation on LDA traverse. DLR's fiber-imaging DGV camera system can be seen mounted on the traverse (left). Right: Inside view of the test section showing ONERA camera system and light sheet generator. The airfoil can be recognized in the middle of the test section with traversable calibration target mounted behind it. 
Configuration A (ONERA)

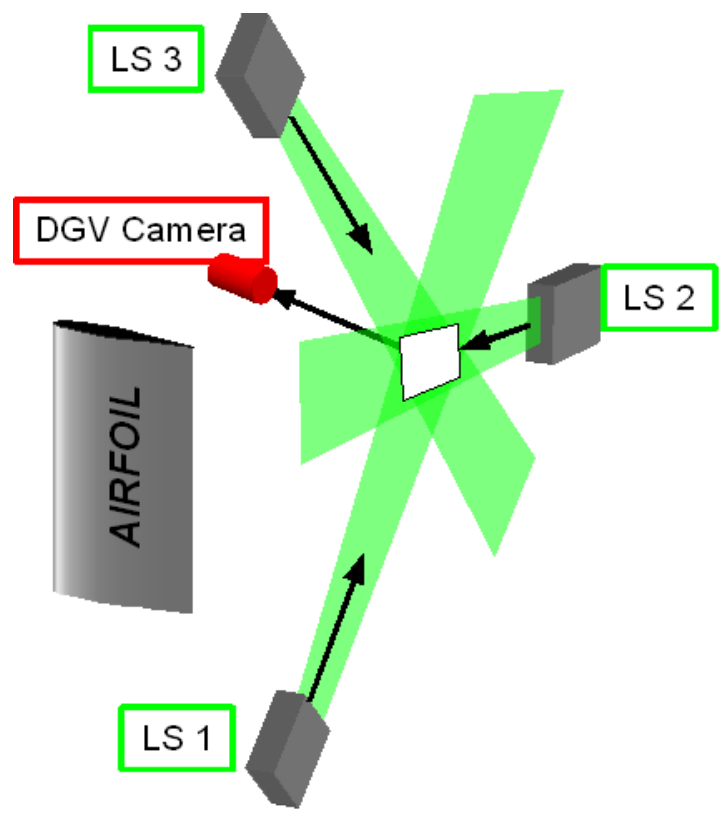

Configuration B (DLR)

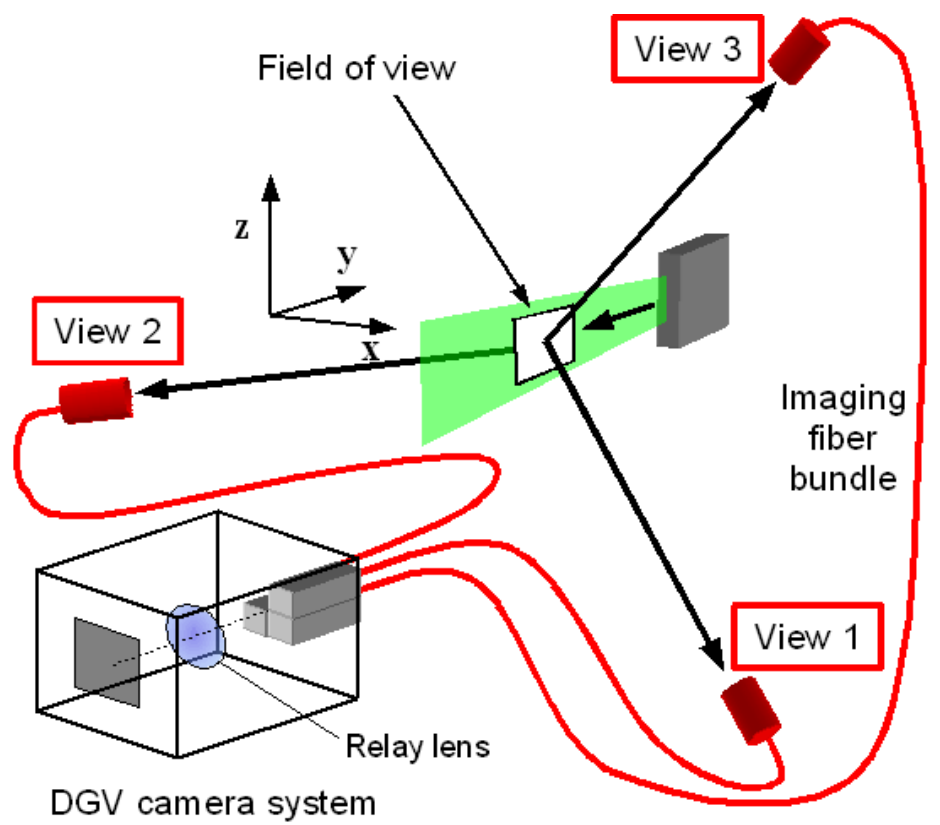

Fig. 5 DGV light sheet and imaging configurations applied in this investigation. Configuration A: Triple light sheet single camera setup used with ONERA camera; Configuration B: Single light sheet - triple camera setup used by DLR.

\subsection{DGV imaging configurations and sensitivity analysis}

As shown in Fig. 4 and Fig. 5 the coplanar laser light sheets can be introduced from three different directions. Given a single viewpoint, a set of three Doppler signal images - one for each light sheet direction - is sufficient to reconstruct the three-component velocity vector map of the flow field. This imaging configuration, shown in Fig. 5 (left), was used by the directly imaging camera system (View 4). On the other hand the fiber imaging camera system principally has the possibility of reconstructing the three-component flow field from any triple combination of light sheet and viewing directions, provided non-co-linearity of the Doppler sensitivity vectors. Nonetheless a configuration using all three view points (Views 1,2,3) and a single light sheet (LS2) was of primary interest for this investigation (Fig. 5, right).

The velocity sensitivity of any DGV imaging configuration can be determined through calculation of the respective sensitivity vectors which in turn are linearly related to the vector difference $\left(\boldsymbol{o}_{\mathrm{i}}-\boldsymbol{l}_{\mathrm{j}}\right)$ between observation $\left(\boldsymbol{o}_{\mathrm{i}}\right)$ and light sheet vectors $\left(\boldsymbol{l}_{\mathrm{j}}\right)$. Decomposition into the three orthogonal directions of the wind tunnel coordinate system provides an estimate for the relative uncertainty of each velocity component. Table 1 summarizes the measurement uncertainties for various imaging configurations assuming a fixed uncertainty in the Doppler frequency measurements $( \pm 1 \mathrm{MHz})$ provided by the camera systems. In spite of the completely different imaging configurations the estimated measurement uncertainties are only slightly different, which has two main reasons: (1) the angular separation between the light sheets used by the ONERA configuration is similar to the angular separation between the viewing directions on the DLR setup. (2) Except for the backscattering view of light-sheet LS2 by the ONERA camera system, all lightsheet view combinations share a similar forward scattering angle $\left(\sim 60^{\circ}\right)$ which directly determines the Doppler shift sensitivity. The backscatter view $\left(\boldsymbol{o}_{4}-\boldsymbol{l}_{2}\right)$ has a roughly three times higher Doppler sensitivity and thereby increases the overall measurement accuracy. It should be mentioned here that the decreased light scattering efficiency in backscattering requires a proportionally longer integration time of the camera to achieve similar signal levels as in forward scattering.

Table 1 also lists measurement uncertainties for several other light sheet camera view arrangements of the DLR imaging configuration. Noteworthy here is that the measurement uncertainty can only be significantly decreased if purely backscatter signals are being used. For 
reasons described later the backscatter signals generally tend to be noisier and bias-prone than the forward scatter signals in spite of the fact of providing higher Doppler shifts. The DGV data presented herein rely on the first two configurations presented in Table 1 as well as Fig. 5.

Table 1 Velocity sensitivity analysis for the two DGV imaging setups assuming a frequency uncertainty of $1 \mathrm{MHz}$ (cell transmission uncertainty $\sim 0.25 \%$ ). Scattering angles less than $90^{\circ}$ correspond to forward scattering.

\begin{tabular}{lccccccc}
\hline $\begin{array}{l}\text { Imaging } \\
\text { configuration }\end{array}$ & views & $\begin{array}{c}\text { light- } \\
\text { sheets }\end{array}$ & $\begin{array}{c}\text { DGV } \\
\text { components }\end{array}$ & $\begin{array}{c}\text { scattering angles } \\
\text { LS1 / LS2 } / \mathrm{LS} 3\end{array}$ & $\begin{array}{r}\Delta \mathrm{U} \\
{[\mathrm{m} / \mathrm{s}]}\end{array}$ & $\begin{array}{r}\Delta \mathrm{V} \\
{[\mathrm{m} / \mathrm{s}]}\end{array}$ & $\begin{array}{r}\Delta \mathrm{W} \\
{[\mathrm{m} / \mathrm{s}]}\end{array}$ \\
\hline ONERA & 1 & 3 & $O_{4}-l_{1}, O_{4}-l_{2}, O_{4}-l_{3}$ & $64^{\circ} / 129^{\circ} / 58^{\circ}$ & $\mathbf{0 . 4 9}$ & $\mathbf{0 . 8 0}$ & $\mathbf{0 . 6 1}$ \\
DLR & 3 & 1 & $O_{1}-l_{2}, O_{2}-l_{2}, O_{3}-l_{2}$ & $56^{\circ} / 53^{\circ} / 62^{\circ}$ & $\mathbf{0 . 6 3}$ & $\mathbf{0 . 9 6}$ & $\mathbf{0 . 9 0}$ \\
DLR & 3 & 3 & $O_{1}-l_{3}, O_{2}-l_{1}, O_{3}-l_{1}$ & $140^{\circ} / 118^{\circ} / 138^{\circ}$ & 0.63 & 0.33 & 0.42 \\
DLR & 3 & 1 & $O_{1}-l_{4}, O_{2}-l_{4}, O_{3}-l_{4}$ & $124^{\circ} / 127^{\circ} / 118^{\circ}$ & 0.61 & 0.27 & 0.89 \\
$\begin{array}{l}\text { LS2 reversed) } \\
\text { DLR }\end{array}$ & 1 & 3 & $O_{1}-l_{1}, O_{1}-l_{2}, O_{1}-l_{3}$ & $94^{\circ} / 56^{\circ} / 140^{\circ}$ & 1.09 & 0.49 & 0.80 \\
DLR. & 1 & 3 & $O_{2}-l_{1}, O_{2}-l_{2}, O_{2}-l_{3}$ & $118^{\circ} / 53^{\circ} / 118^{\circ}$ & 0.72 & 0.49 & 0.80 \\
DLR. & 1 & 3 & $O_{3}-l_{1}, O_{3}-l_{2}, O_{3}-l_{3}$ & $138^{\circ} / 62^{\circ} / 91^{\circ}$ & 1.08 & 0.49 & 0.80 \\
$\begin{array}{l}\text { Generic case } \\
\left(120^{\circ} \text { between LS) }\right.\end{array}$ & 1 & 3 & - & $90^{\circ} / 90^{\circ} / 90^{\circ}$ & $\mathbf{0 . 5 9}$ & $\mathbf{0 . 5 9}$ & $\mathbf{0 . 4 1}$ \\
\hline
\end{tabular}

\section{Data acquisition and calibration}

Reconstruction of the Cartesian three-component velocity vector map from the Doppler shift image triplets requires a precise calibration of the camera positions with respect to the field of view to define the local observation vectors $\left(\boldsymbol{o}_{\mathrm{i}}\right)$. Similarly maps of the spatially calibrated light incidence vectors $\left(\boldsymbol{l}_{\mathbf{j}}\right)$ must be determined. The estimation of the camera positions relies on camera calibration techniques common in machine vision using images of known calibration targets (Tsai 1987, Willert 2006). In this case a dual-sided planar target with a regular grid of dots $\left(10 \times 10 \mathrm{~mm}^{2}\right.$ spacing, $2 \mathrm{~mm}$ diameter) was traversed normally to the light sheet plane at $6 \mathrm{~mm}$ intervals. Calibration was performed using up to five images for each view, accounting for the target thickness in View 2. Given images of the type shown in Fig. 6 (left) the variance in the recovered camera positions seems to be more or less independent of the choice of camera model and indicates an angular viewing uncertainty on the order of $0.1^{\circ}$ (Willert 2006). The influence of this angular error is well below that of other error sources.

The calibration data provided by the target images was also used to calculate image mapping functions for each view resulting in a common reconstructed image space. Light sheet incidence angles were determined from the reconstructed images of striped light sheet images using manual procedures or automated line detection schemes based on the Hough transformation.

With the geometrical calibration completed actual DGV image acquisition could commence. In a first step a set of background images in the absence of seeding was acquired. Then stream tube seeding slowly filled the wind tunnel and DGV images of the type shown in Fig. 6 (middle) were acquired. The strong forward scattering efficiency of the seeding droplets allowed exposure times as low as $250 \mathrm{~ms}$ while stream tube seeding was active. The backscatter signal was roughly 5-10 lower which required a proportional increase in the camera exposure time. After several minutes of continuous seeding the tunnel was sufficiently seeded such that very uniform light sheet images of the type shown in Fig. 6 (right) could be acquired. Compared to the stream tube seeding the intensity levels of this global seeding were roughly three times lower requiring a proportional 
increase of the camera exposure times (1-3 seconds). The seeding was observed to be persistent over a several hours indicating a low tendency toward evaporation or coagulation as well as a small droplet diameter (e.g. low settling velocity).

For each wind tunnel set point several image sets were acquired in parallel by the two camera systems. Aside from repeating each measurement at least twice, variations of exposure times and seeding conditions were performed. In a final step of each measurement sequence the laser was detuned to the $100 \%$ transmission plateau of the iodine cell such that flat field images could be collected which are required to normalize the intensity ratio images.
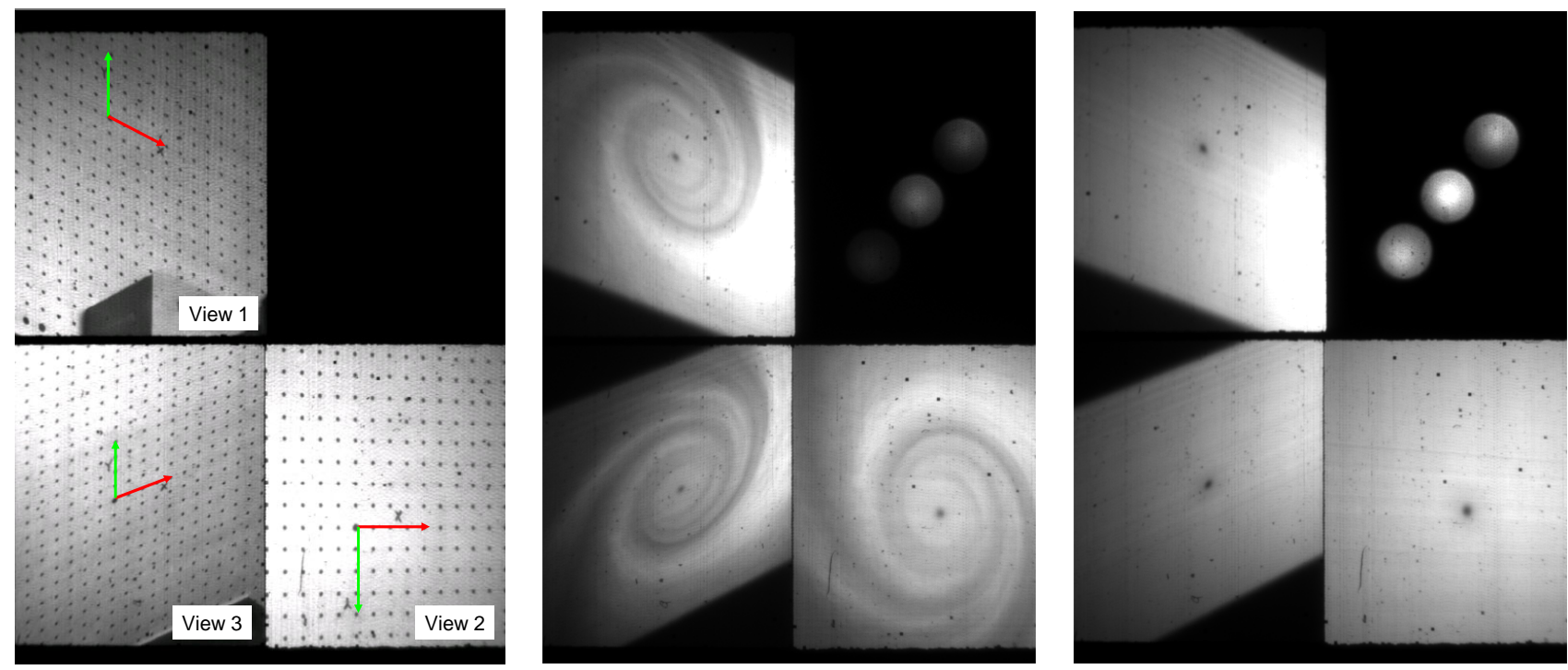

Fig. 6 Images acquired with the multiple branch fiber imaging bundle DGV camera system: calibration target (left), light sheet LS2 illuminating wake vortex with stream tube seeding (middle), same wake vortex under ambient seeding conditions (right). Circular dots in upper right provide laser reference frequencies at $-80,0,+80 \mathrm{MHz}$.

\section{Results}

Two principle experiments were performed on two separate days. In one case the imaged plane was traversed in the stream-wise $(x)$ direction to map the evolution of the wake vortex; in the other case the airfoil's angle of attack was varied. Fig. 7 presents data for the reference condition at a free stream velocity of $U_{\infty}=68 \mathrm{~m} / \mathrm{s}, x=450 \mathrm{~mm}$ downstream of the wingtip at angel of attack $\alpha=8.5^{\circ}$ for which corresponding LDA is available (Fig. 3). The color lookup table for the out-of-plane component, $U$, is purposely chosen to bring out variations on the order of $1-2 \mathrm{~m} / \mathrm{s}$. Profiles extracted from these data sets - given in Fig. 8 - show very good agreement on the order of $1 \mathrm{~m} / \mathrm{s}$. Nonetheless the data obtained by the fiber imaging system (DLR) has the tendency of smoothing out velocity gradients which is a direct consequence of the reduced spatial resolution of this system. Also spatial averaging has been performed on the data to reduce noise and high-frequency artifacts introduced by the regular structure of the fiber imaging bundles. Without smoothing the noise amplitudes are on the order of $\pm 2 \mathrm{~m} / \mathrm{s}$ (see also Fig. 11). The data obtained by the single-view DGV system (ONERA) shows an even better agreement with the LDA data slightly underestimating the radial velocity $W$. This underestimation believed to be caused by multiple scattering between the particles especially in densely seeded flows which tends to smooth velocity gradients.

The evolution of the vortex structure for three different axial positions downstream of the airfoil is shown in Fig. 9 as obtained by DLR's fiber imaging bundle system. On the whole little variation on the wake vortex can be observed, except for a low speed packet of fluid that orbits around the vortex core. 

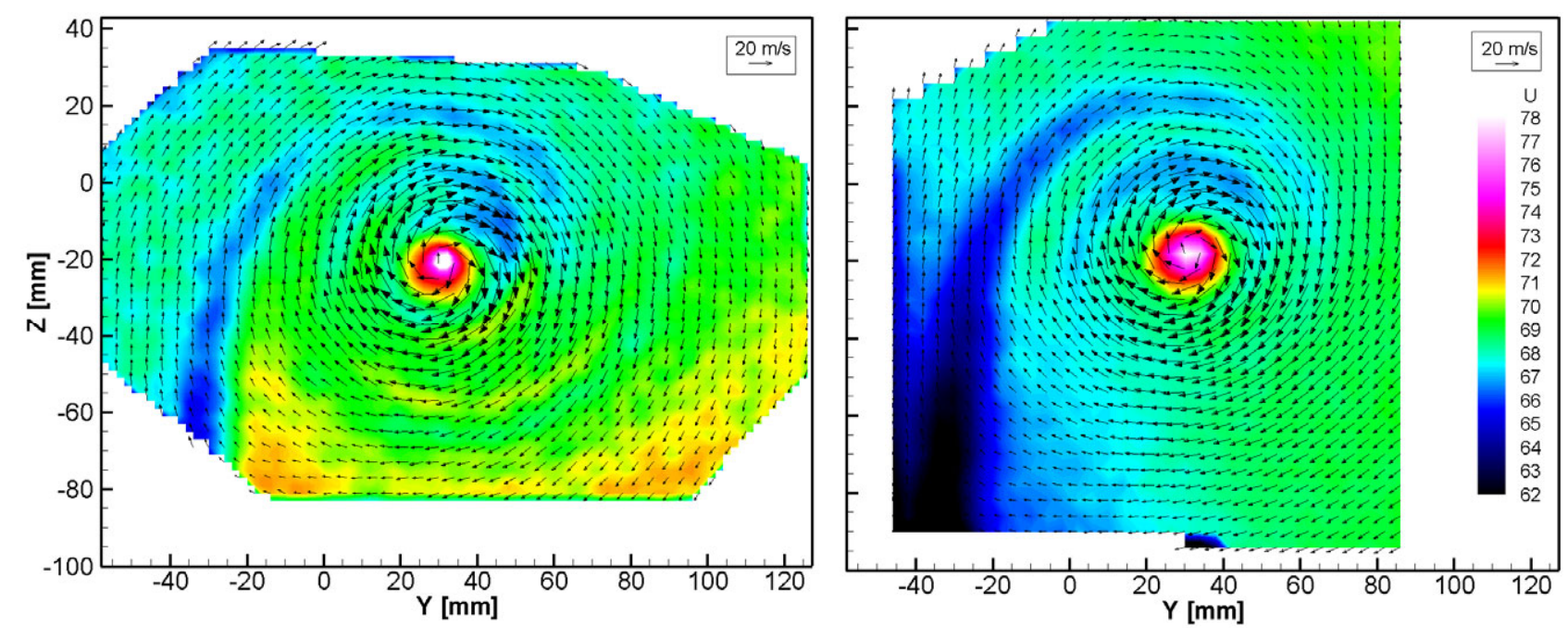

Fig. 7 Flow field measurements obtained at $U_{\infty}=67 \mathrm{~m} / \mathrm{s}$ and $\alpha=8.5^{\circ}$ by ONERA-DGV configuration (left) and DLR configuration (right).
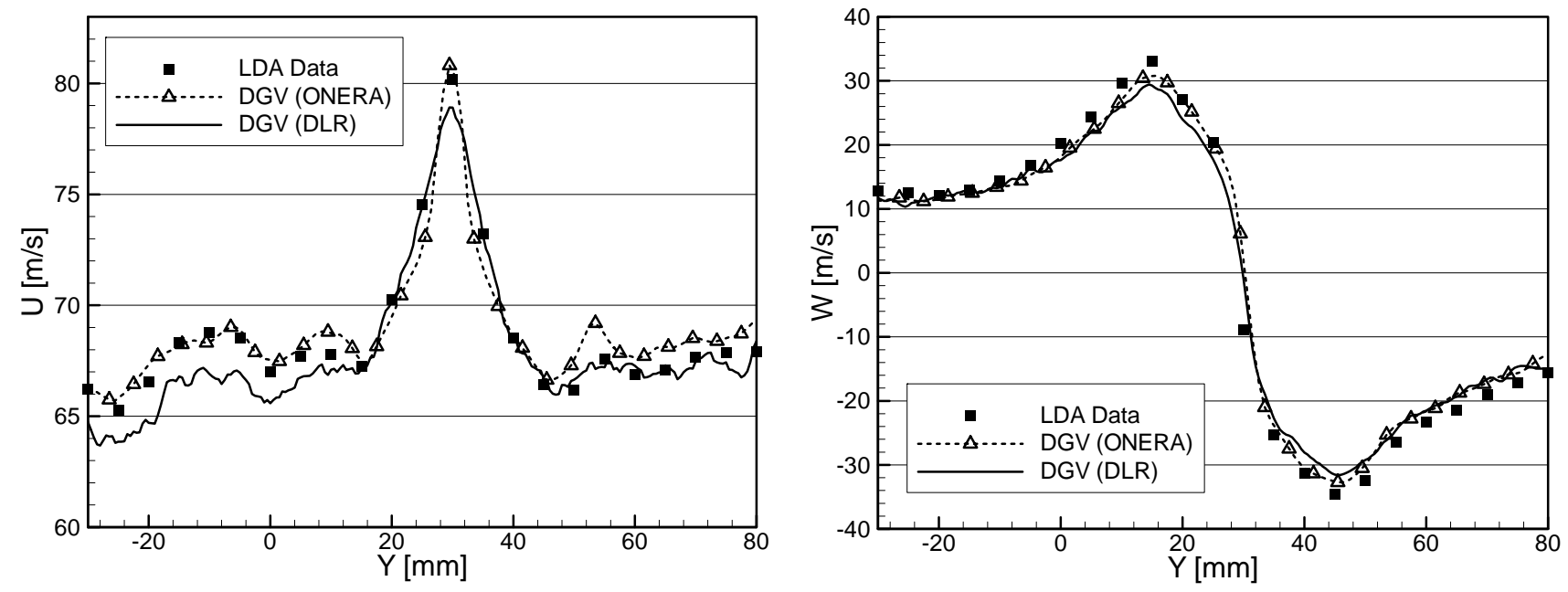

Fig. 8 Comparison between DGV and LDA velocity data for axial component ( $U$, left) and vertical component (W, right) through the vortex core along the $Y$-direction.

More striking variations of the flow field are visible when the airfoil's angle of attack is varied (Fig. 10). At zero incidence a small vortex structure can be seen above the main wake. It has inplane velocity amplitudes around $3 \mathrm{~m} / \mathrm{s}$ which correspond to Doppler frequency variations on the order of $5 \mathrm{MHz}$. With increasing angle of attack this vortex structure is entrained into the forming tip vortex. The core flow exhibits a flow reversal between $5.5^{\circ}$ and $7^{\circ}$.

The noise in the Doppler shift images acquired by the fiber imaging camera system is around $\pm 2 \mathrm{MHz}$ and is mainly due to fiber bundle artifacts. The direct viewing camera system was able to achieve noise levels that were about four times lower. While both systems achieve similar spatial resolutions $(0.5-1 \mathrm{~mm})$, it should be remembered that the fiber bundle system images the same field of view onto three quadrants of the sensor which already reduces the signal-to-ratio by a factor of two in comparison to a single recording filling the available sensor space.

The data acquired by the fiber imaging bundle system exhibits a velocity gradient which can be seen quite clearly in the form of a left-to-right variation in the outer flow in the zero-angle of attack measurement of Fig. 10. Measurements of the quiescent flow (Fig. 11) confirm this to be on the order of $\pm 2 \mathrm{~m} / \mathrm{s}$ across the field of view. The main reason for this is believed to be the illumination of the background scene with the light scattered from the laser light sheet. Background images which are normally acquired prior to the measurement in the absence of seeding do not account for 
this light. A similar effect was observed with measurements performed in a cryogenic wind tunnel facility (Willert et al. 2005). The influence of this stray light could have been reduced by placing light-absorbent materials (e.g. black felt, flat black paint) in the scene background. This was done for the ONERA camera system.

A significant amount of noise in the measurements obtained by the multiple view camera system is caused by irregularities and voids in the fiber imaging bundles. Even with appropriate spatial filtering and image registration in the sub-pixel range, this noise cannot be completely removed. A laser-line filter placed inside the camera system was later found to cause spatial smoothing of gradients due to light reflected back onto the fiber bundle. A placement at a different location inside the camera system solves this problem. The spatial resolution is also somewhat compromised by a rotating half-wave plate placed in front of the beam splitter which causes some lateral beam displacement. Here a wedge-depolarizer, as used by the single view camera system, seems to provide equally good results.
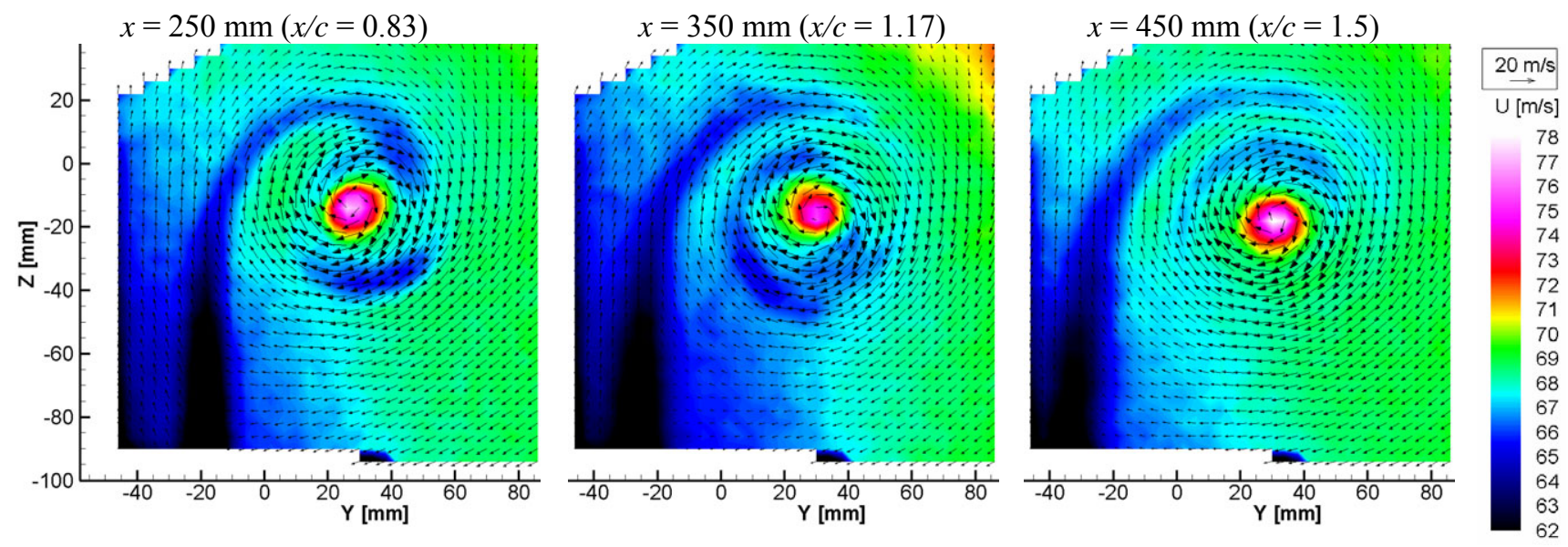

Fig. 9 Development of the tip vortex downstream of the airfoil
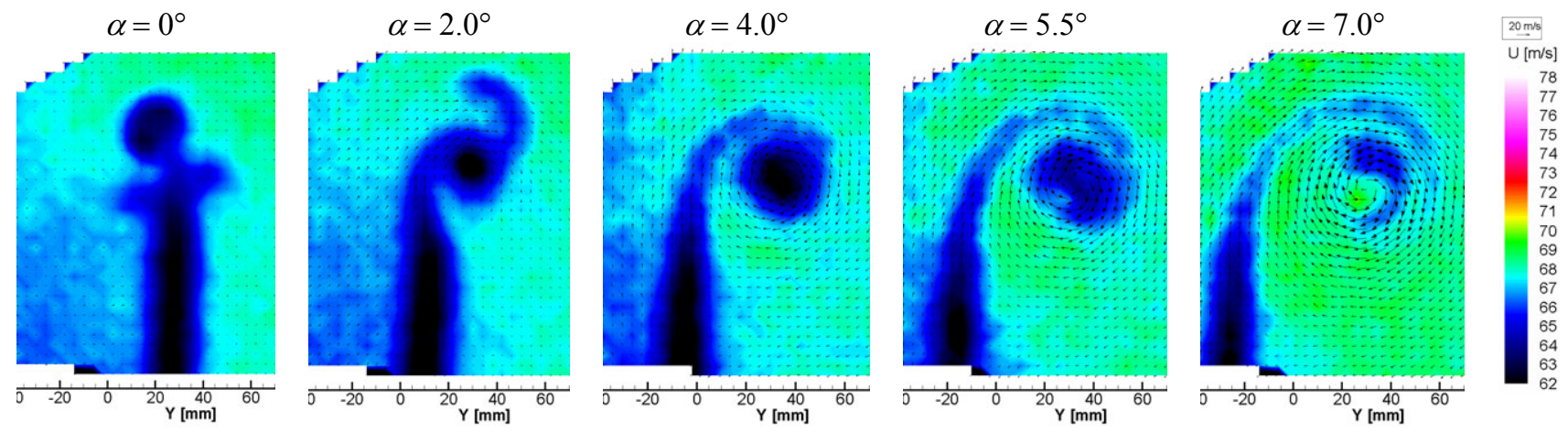

Fig. 10 Development of vortex with increasing angle of attack at $x=450 \mathrm{~mm}$ downstream of airfoil.

\section{Conclusions}

The described measurement campaign provided the unique opportunity of simultaneously operating two DGV systems allowing not only a side-by-side performance comparison but also allowing a direct bench-marking against a reference data set provided by LDA measurements. The agreement between the various techniques is within 1-2 $\mathrm{m} / \mathrm{s}$, with DGV slightly underestimating local velocity gradients. As to be expected by the better imaging quality of the direct viewing camera system provided by ONERA, the results obtained by this system are characterized by less noise than the data acquired by the multiple view camera system provided by DLR which relies on fiber imaging bundles. The main advantage of the multiple view fiber imaging system is that a 
single recording captures the complete flow field using only a single light sheet and therefore requires only one third of the acquisition time.

In terms of sensitivity, which depends on the respective viewing and light sheet geometries, both systems achieve similar velocity sensitivities. While the multiple view system made use of increased signal levels by observing the light sheet in forward scatter, the single view system had to observe one light sheet in backscatter, which increased the measurement time but also increased the Doppler shift sensitivity for this view which in turn resulted in slightly better velocity sensitivity. It should be observed that the overall measurement noise is dominated by the Doppler shift image with the lowest signal-to-noise ratio, which implies that all three Doppler shift images should be of similar quality. A more detailed sensitivity analysis on the background of DGV implementation in wind tunnel environments is given by Lempereur et al. (2006).

Just as concluded in earlier DGV measurement campaigns the importance of background light suppression is not to be underestimated. Most of the velocity biasing observed in the current tests can be attributed to this light, which cannot be removed simply by recording background images in the absence of flow seeding. Aside from properly covering the scene background with light absorbent materials (e.g. black felt), a possible solution to the problem may be the use of techniques that are capable of obtaining the background signal during the actual measurements. One such method is currently being developed at DLR and relies on acquiring striped light sheets in addition to the standard DGV light sheet images. The background can be extracted from the regions between the laser stripes, with images of the complementary stripes filling the remainder. Clearly this method is restricted to time-averaged velocity measurements, but it particularly useful in environment where background luminosity is significant (e.g. combustion chambers).

With regard to DGV imaging geometry in general, none of the two setups is particular superior to the other as both require access from four points, either one camera and three light sheets or three camera views and one light sheet. The only possibility to reduce this geometrical complexity is to combine the DGV technique with a different method such as PIV in which case only one light sheet port and one camera view port would be required (Wernet 2005; Willert et al. 2006). Nonetheless the fiber based imaging system has the potential of being the least intrusive requiring only small access ports with minor space requirements (typically only a small lens with support).

While the joint measurement campaign pointed to a wide variety of possible improvements of the DGV method, the possibility of using it for online analysis as proposed in the original patent of Komine 1990 to date has not been fully explored. During a demonstration performed in front of the audience of a workshop on the subject, live DGV measurements were demonstrated. In effect Doppler shift images can be produced online allowing a real-time investigation of complex flow fields and therefore could be used to identify flow conditions which are worthwhile for analysis by more precise and complex measurement techniques.

\section{Acknowledgments}

Support for this joint ONERA/DLR measurement campaign was provided by the European Windtunnel Association (EWA), a Network of Excellence within the $6^{\text {th }}$ Framework Programme funded by the European Commission ( http://www.eu-ewa.areo ).

\section{References}

Barricau P, Lempereur C, Mathé JM, Mignosi A, Buchet H (2002) Doppler global velocimetry: development and wind tunnel tests. Proc: Application of Laser Techniques to Fluid Mechanics, Lisbon

Elliot GS, Beutner TJ (1999) Molecular filter based planar Doppler velocimetry. Prog. Aero Sci 35:799-845

Komine H (1990) System for measuring velocity field of fluid flow utilizing a laser-Doppler spectral image converter. US patent no. 4,919,536

Lempereur C, Barricau P, Gleyzes C, Willert C, Stockhausen G, Klinner J (2006) Doppler global 
velocimetry in wind tunnels: implementation issues and performance analysis. Proc: Application of Laser Techniques to Fluid Mechanics, Lisbon

Samimy M, Wernet MP (2000) Review of planar multiple-component velocimetry in high-speed flows. AIAA Journal 38:553-574

Nobes D, Ford H, Tatam R (2004) Instantaneous, three component planar Doppler velocimetry using imaging fibre bundles. Exp Fluids 36:3-10

Tsai R.Y. (1987) A versatile camera calibration technique for high-accuracy 3D machine vision metrology using off-the-shelf TV cameras and lenses. IEEE J. Robot. Autom. RA-3:323-344

Wernet P (2004) Planar particle imaging Doppler velocimetry: a hybrid PIV/DGV technique for threecomponent velocity measurements. Meas Sci Technol 15:2011-2028

Willert C (2005) Doppler global velocimetry - Fundamentals, imaplementation and selected applications. Lecture Series 2005-01: Advanced Measurement Techniques for Supersonic Flows, von Karman Institute of Fluid Dynamics, Brussels

Willert C, Stockhausen G, Beversdorff M, Klinner J, Lempereur C, Barricau P, Quest J, Jansen U (2005) Application of Doppler global velocimetry in cryogenic wind tunnels. Exp Fluids 39:420-430

Willert C, Hassa C, Stockhausen G, Jarius M, Voges M, Klinner J (2006) Combined PIV and DGV applied to a pressurized gas turbine combustion facility. Meas Sci Technol (to appear)

Willert C (2006) Assessment of camera models for use in planar velocimetry calibration. Exp Fluids (to appear)
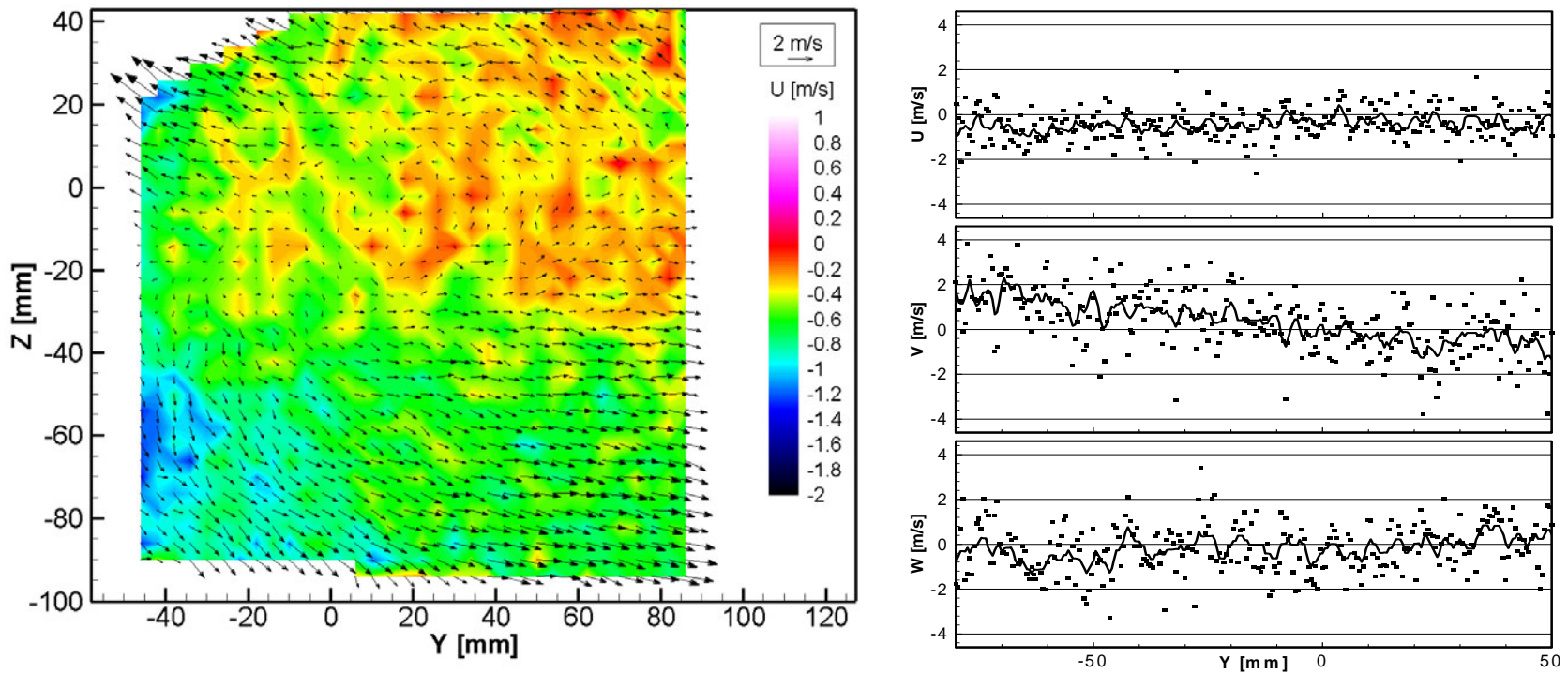

Fig. 11 Velocity map of the 'zero-flow' condition showing the influence of stray background illumination. Right: Velocity profiles along $y=10 \mathrm{~mm}$ for unfiltered (symbols) and spatially filtered data (solid line). 